\title{
Bouchout's Tuberculoma
}

\author{
Mehdi Khamaily, Wafae Akioud, Joumany Brahim Salem, Sidi Dahi, Houda Brarou, \\ Taoufik Abdellaoui, Yassine Mouzari, Karim Reda, Abdelbarre Oubaaz
}

\section{ABSTRACT}

Tuberculosis is a chronic infection that is still endemic in Morocco. Eye involvement, however, is quite rare. We report the discovery of Bouchut's tuberculoma in a 52-year-old immunocompetent patient following a progressive decline in visual acuity in both eyes.

Keywords: Tuberculosis, Posterior uveitis, anti-bacillary.
Published Online: September 15, 2020

ISSN: $2593-8339$

DOI: $10.24018 /$ ejmed.2020.2.5.485

Mehdi Khamaily

Mohammed V Military Teaching Hospital, Morocco.

(e-mail: mehdi.khamaily@ gmail.com)

Wafae Akioud

Mohammed V Military Teaching Hospital, Morocco.

Joumany Brahim Salem

Mohammed V Military Teaching Hospital,

Morocco.

Sidi Dahi

Mohammed V Military Teaching Hospital,

Morocco.

Houda Brarou

Mohammed V Military Teaching Hospital, Morocco.

Taoufik Abdellaoui

Mohammed V Military Teaching Hospital,

Morocco.

Yassine Mouzari

Mohammed V Military Teaching Hospital,

Morocco.

Karim Reda

Mohammed V Military Teaching Hospital,

Morocco.

Abdelbarre Oubaaz

Mohammed V Military Teaching Hospital, Morocco.

*Corresponding Author

\section{INTRODUCTION}

Tuberculosis is a chronic infection caused by Mycobactrium tuberculosis ( MT), that is still endemic in Morocco. Eye involvement, however, is quite rare with very variable clinical pictures.

\section{CASE REPORT}

We report the case of a 52-year-old male patient with no specific medical history, in particular no notion of tuberculosis contagion or immunosuppression, presented for an ophthalmologic consultation for a progressive decrease of the visual acuity more pronounced on the right eye.

The ophthalmologic examination found a visual acuity corrected to 10/20 right eye, and 14/20 left eye. In addition, no abnormalities of the appendages or the anterior segment were noted, and this in both eyes.

The Fundus examination finds multiple deep buff-yellow lesions predominantly in the posterior pole of the right eye juxta-macular as well as some lesions of the posterior pole in the left eye. The vitreous is normal. Systemic examination does not find any abnormalities that could suggest extraocular tuberculosis.

The Retinal fluorescein angiography shows multiple deep lesions (cockade appearance) juxta macular early in the right eye, becoming hyper fluorescent later in life. The left eye shows a few scattered hyperfluorescent lesions(Fig. 1, 2) No lesion of vasculitis or papillitis is noted. The patient received a positive physiologic assessment and a negative HIV serology.

The patient is put on antibacillary treatment combining Streptomycin, Rifampicin, Isoniazid, and Pyrazinamide for 2 months, then a combination of Rifampicin and Isoniazid for 7 months. Systemic corticosteroid therapy based on dexamethasone at a dosage of $1 \mathrm{mg} / \mathrm{kg} /$ day is prescribed. The evolution under treatment is favorable. 

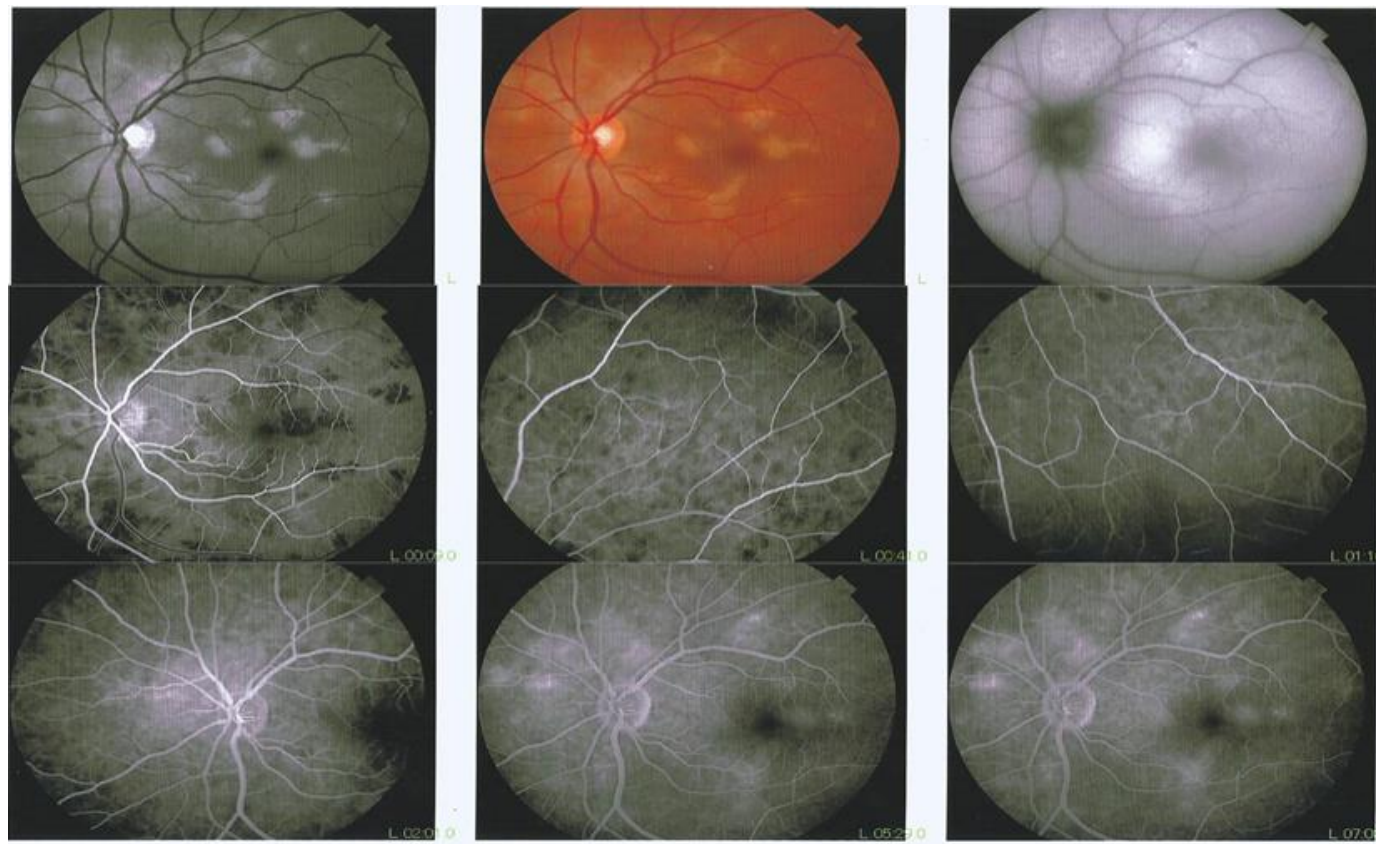

Fig. 1. Fluorescein angiogram showing juxta-macular lesions in front of hyperfluorscent at late stages in both eyes.
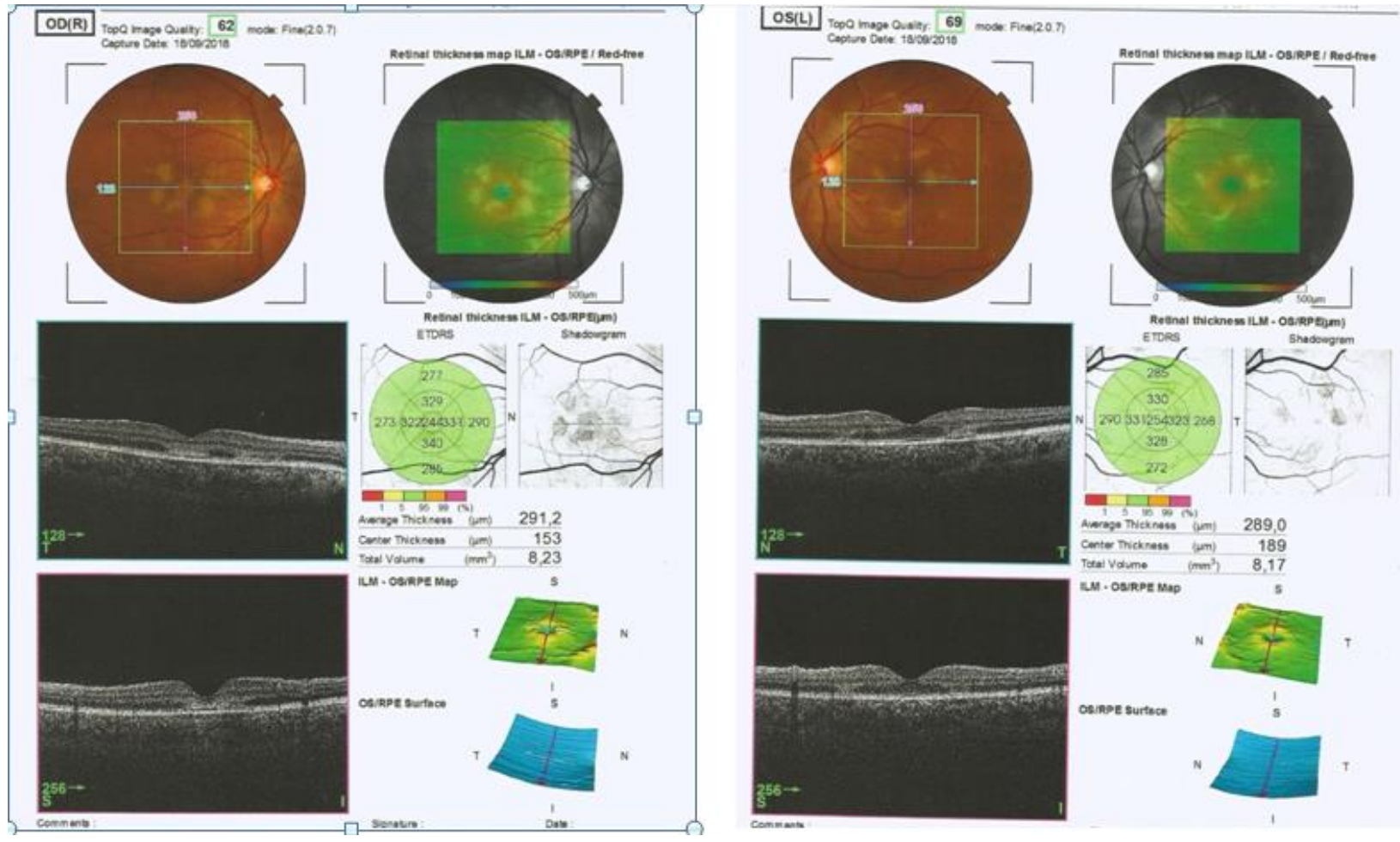

Conte Tickness (4) 189

Thivive

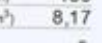

Fig. 2. Optical Coherence Tomography : Peri-macular infiltrates in both eyes.

\section{DISCUSSION}

Tuberculosis is a major cause of morbidity and mortality, with an estimated prevalence of around 25,000 new cases / year [1]. The extra-pulmonary locations of tuberculosis represent $35 \%$ of all cases, including 1 to $2 \%$ are ocular damage [2], [3].

Some conditions such as Bouchut's tuberculoma are linked to the presence of Koch's bacilli in the eye, others such as uveitis are a reflection of delayed hypersensitivity to these bacilli [4].

Clinically, tuberculous choroiditis is characterized by the classic Bouchut tubercles which are buff-yellow nodules, mainly located at the posterior pole. These nodules are unilateral in $80 \%$ of cases [5].

Fluorescein retinal angiography is of great interest for diagnostic workup [6].

The anti-tuberculosis treatment is well codified by recent recommendations and begins with a quadritherapy induction for 2 months then a dual consolidation therapy for a period of 7 to 10 months associated with corticosteroid therapy for 8 weeks [7]. 
European Journal of Medical and Health Sciences www.ejmed.org

\section{CONCLUSION}

Tuberculosis continues to be rampant in an endemic state in Morocco, taking various presentations such as ocular involvement, hence the interest of a systematic ophthalmological examination in case of suspicion of tuberculosis.

\section{REFERENCES}

[1] Wani S. R., Wattal C., Raveendran R. "Epidemiology and risk factors associated with NTM pulmonary and extrapulmonary infections in a high tuberculosis endemic Region", Indian J Med Microbiol, 2020; vol. 38, pp. 169-75.

[2] Sirang, Z. (2020)." Intra-ocular Tuberculosis: controversies regarding diagnosis and treatment", Nepalese Journal of Ophthalmology, vol. 12(1), pp. 158-161. https://doi.org/10.3126/nepjoph.v12i1.23044.

[3] Bisht D., Pande R., "Study of ocular manifestations in tuberculosis and its association with HIV AIDS in a tertiary care hospital", Indian Journal of Tuberculosis, https://doi.org/10.1016/j.ijtb.2019.10.004.

[4] Ramanjulu R., Dubey D., Shanmugam M. P. "Simultaneous mutually exclusive active tubercular posterior uveitis", Indian J Ophthalmol, 2020, vol. 68, pp. 2049-50.

[5] Salil Mehta, Remco PH Peters, Derrick P. Smit \& Vishali Gupta (2020), "Ocular Tuberculosis in HIV-infected Individuals", Ocular Immunology and Inflammation, DOI 10.1080/09273948.2020.1776882.

[6] Herbort, C. P., Tugal-Tutkun, I., Mantovani, A. et al. "Advances and potential new developments in imaging techniques for posterior uveitis Part 2: invasive imaging methods", Eye, (2020). https://doi.org/10.1038/s41433-020-1072-0.

[7] Testi I., Agrawal R., Mehta S., Basu S., Nguyen Q., Pavesio C. Gupta V. "Ocular tuberculosis: Where are we today?", Indian J Ophthalmol, 2020, vol. 68, pp. 1808-17. 